\title{
Pseudo almost periodic weak solutions of a semilinear elliptic equation
}

\author{
Desheng $\mathrm{Ji}^{1 *}$ and Chuanyi Zhang ${ }^{2}$
}

\author{
"Correspondence: dji@hit.edu.cn \\ 1 Department of Mathematics, \\ Harbin Institute of Technology, West \\ Dazhi Street, Harbin, 150001, China \\ Full list of author information is \\ available at the end of the article
}

\begin{abstract}
In this paper, pseudo almost periodic functions on $\mathbb{R}^{N}$, with $N$ an integer larger than 1 , are introduced and some basic properties of them are studied. As an application, we investigate the pseudo almost periodicity of a weak solution of the semilinear elliptic equation $-\Delta u+\sum_{j=1}^{N} c_{j} \partial_{j} u+f(x, u)=h(x)$. In addition, a pseudo almost periodic forced pendulum equation is considered as an example.

MSC: Primary 35B15; secondary 35J61

Keywords: almost periodicity; pseudo almost periodicity; semilinear elliptic equation
\end{abstract}

\section{Introduction}

The notion of pseudo almost periodic functions on $\mathbb{R}$ was introduced by Zhang [1-3] in the early 1990s and it is a natural generalization of Bohr almost periodicity. In the past 20 years, these functions have attracted much attention and have been applied to qualitative analysis for various kinds of equations (see [4-8] and the references therein).

The notion of Bohr almost periodicity is also suitable for functions on $\mathbb{R}^{N}$, with $N>1$. This notion was deeply studied for numerical-valued functions in Bochner's fundamental paper [9]. In early 1970s, Sibuya [10] and Sell [11] investigated almost periodic solutions of some linear partial differential equations. Then Zaidman [12] studied almost periodic functions from $\mathbb{R}^{N}$ into a Banach space. Recent work on almost periodic second order elliptic equations in $\mathbb{R}^{N}$ (see, for instance, Pankov [13], Fournier et al. [14] and N'Guérékata [15]) shows a revival of the interest in this topic.

It is a natural idea to introduce pseudo almost periodic functions on $\mathbb{R}^{N}$ and these functions may have potential use in differential equations with more than one spatial variables. However, to the best of our knowledge, there is no literature systematically treating this yet. So in this paper, we will introduce these functions and study some of their basic properties. To illustrate their potential use, we also study the pseudo almost periodicity of a weak solution of the following semilinear elliptic equation:

$$
-\Delta u+\sum_{j=1}^{N} c_{j} \partial_{j} u+f(x, u)=h(x), \quad x \in \mathbb{R}^{N},
$$

where $c_{j}$ 's are real constants, $f$ and $h$ are pseudo almost periodic functions.

\section{Springer}

(O2014 Ji and Zhang: licensee Springer. This is an Open Access article distributed under the terms of the Creative Commons Attribution License (http://creativecommons.org/licenses/by/2.0), which permits unrestricted use, distribution, and reproduction in any medium, provided the original work is properly cited. 
In order to study (1.1), we need to consider its almost periodic component equation at first, i.e.,

$$
-\Delta v+\sum_{j=1}^{N} c_{j} \partial_{j} v+g(x, v)=h_{1}(x), \quad x \in \mathbb{R}^{N},
$$

where $g$ and $h_{1}$ are the almost periodic components of $f$ and $h$, respectively.

For the existence of bounded weak solutions of equations (1.1) and (1.2), we refer to the work of Fournier et al. [14]. Our main task is the following two steps.

- We firstly show the almost periodicity of a bounded weak solution $v$ of (1.2). This result is an improvement of [14, Theorem 3.2] where $g(x, v)=g(v)$.

- Then, we show that the bounded weak solution $u$ of (1.1) is pseudo almost periodic by proving that $u-v$ is an ergodic perturbation.

The organization of this paper is as follows. In Section 2 we recall some basic facts on Bohr almost periodic functions on $\mathbb{R}^{N}$. Section 3 is devoted to the pseudo almost periodic functions on $\mathbb{R}^{N}$. In the last section, we investigate pseudo almost periodicity of a weak solution of (1.1) and consider a pseudo almost periodic forced pendulum equation as an example.

\section{Bohr almost periodic functions}

Let $E=\mathbb{R}$ or $\mathbb{C}, E^{n}$ be the $n$-dimensional real or complex Euclidean space with norm $|x|=$ $\left(\sum_{i=1}^{n} x_{i} \overline{x_{i}}\right)^{1 / 2}$ and production $x \cdot y=\sum_{i=1}^{n} x_{i} y_{i}$ for any $x, y \in \mathbb{R}^{n}$. Let $N$ be an integer larger than 1 . A closed ball in $\mathbb{R}^{N}$ (of center $x_{0}$ and radius $\ell>0$ ) is defined by $B\left(x_{0}, \ell\right)=\left\{x \in \mathbb{R}^{N}\right.$ : $\left.\left|x-x_{0}\right| \leq \ell\right\}$. The cubes in $\mathbb{R}^{N}$ are defined by $K_{r}=\left\{x \in \mathbb{R}^{N}:\left|x_{j}\right| \leq r, j=1,2, \ldots, N\right\}$ and $s+K_{r}=\left\{s+x: x \in K_{r}\right\}$ where $s \in \mathbb{R}^{N}$. We denote the Lebesgue measure of a set $S \subset \mathbb{R}^{N}$ by $m(S)$.

Let $X$ be a Banach space with norm $\|\cdot\|$. Let $\mathcal{C}\left(\mathbb{R}^{N} ; X\right)$ denote the space of all continuous functions from $\mathbb{R}^{N}$ to $X$ and $\mathcal{B C}\left(\mathbb{R}^{N} ; X\right)$ denote the space of all bounded functions in $\mathcal{C}\left(\mathbb{R}^{N} ; X\right)$. Endowed with the norm

$$
\|f\|_{\infty}=\sup _{x \in \mathbb{R}^{N}}\|f(x)\|
$$

$\mathcal{B C}\left(\mathbb{R}^{N} ; X\right)$ is a Banach space.

We say a set $P \subset \mathbb{R}^{N}$ is relatively dense in $\mathbb{R}^{N}$ if there exists a number $\ell>0$ such that the intersection $P \cap B(x, \ell)$ is non-empty, for all $x \in \mathbb{R}^{N}$.

Definition 2.1 ([12]) A function $f \in \mathcal{C}\left(\mathbb{R}^{N} ; X\right)$ is called almost periodic, if for every positive number $\epsilon$, one may find a relatively dense set $T(f, \epsilon)$ in $\mathbb{R}^{N}$, such that

$$
\|f(\cdot+\tau)-f(\cdot)\|_{\infty} \leq \epsilon, \quad \forall \tau \in T(f, \epsilon) .
$$

We denote by $\mathcal{A P}\left(\mathbb{R}^{N} ; X\right)$ the space of all such almost periodic functions.

$\mathcal{A P}\left(\mathbb{R}^{N} ; X\right)$ contains the space, $\operatorname{Trig}\left(\mathbb{R}^{N} ; X\right)$, which consists of functions $x \mapsto$ $\sum_{j=1}^{n} \exp \left(i \xi_{j} \cdot x\right) v_{j}$ where $v_{j} \in X, \xi_{j}$ and $x \in \mathbb{R}^{N}$, and $n$ is any natural number (see [13, Proposition 1.1.3]). Let $x \in \mathcal{A P}\left(\mathbb{R}^{N} ; X\right)$. Since for each $t, \tau \in \mathbb{R}^{N},|\|x(t+\tau)\|-\|x(t)\|| \leq$ $\|x(t+\tau)-x(t)\|$, it is easy to see that the function $\|x(\cdot)\| \in \mathcal{A P}\left(\mathbb{R}^{N} ; \mathbb{R}\right)$. 
Lemma 2.2 ([12, Chapter 9])

(1) $\mathcal{A} \mathcal{P}\left(\mathbb{R}^{N} ; X\right)$ is a Banach space.

(2) For any $f \in \mathcal{A P}\left(\mathbb{R}^{N} ; X\right)$, the range $\mathcal{R}_{f}=\left\{f(x): x \in \mathbb{R}^{N}\right\}$ is relatively compact.

(3) If $\in \mathcal{A P}\left(\mathbb{R}^{N} ; X\right)$, then it is uniformly continuous over $\mathbb{R}^{N}$.

(4) $f \in \mathcal{C}\left(\mathbb{R}^{N} ; X\right)$ is almost periodic if and only if it is normal; that is, the set $\left\{f(\cdot+s): s \in \mathbb{R}^{N}\right\}$ is relatively compact in $\mathcal{B C}\left(\mathbb{R}^{N} ; X\right)$.

(5) Iff and $g$ both are almost periodic on $\mathbb{R}^{N}$, then for each $\epsilon>0$, the set $T(f, \epsilon) \cap T(g, \epsilon)$ is relatively dense in $\mathbb{R}^{N}$.

Lemma 2.3 ([13, p.10])

(1) For $f \in \mathcal{A} \mathcal{P}\left(\mathbb{R}^{N} ; X\right)$, then

$$
M(f)=\lim _{r \rightarrow \infty} \frac{1}{(2 r)^{N}} \int_{s+K_{r}} f(x) \mathrm{d} x,
$$

where the limit exists uniformly in $s \in \mathbb{R}^{N}$.

(2) Let $f \in \mathcal{A P}\left(\mathbb{R}^{N} ; \mathbb{R}\right)$. If $f \geq 0$ and $M(f)=0$, then $f \equiv 0$.

The representation (2.1) has some other useful versions. For example,

$$
M(f)=\lim _{r \rightarrow \infty} \frac{1}{m(B(s, r))} \int_{B(s, r)} f(x) \mathrm{d} x
$$

uniformly in $s \in \mathbb{R}^{N}$. For the proof we refer the reader to [16].

Let $n$ be a positive integer and $\Omega$ be a subset of $E^{n}$. We denote by $\mathcal{C}\left(\mathbb{R}^{N} \times \Omega ; X\right)$ the set of all jointly continuous functions from $\mathbb{R}^{N} \times \Omega$ to $X$. We give the following definition.

Definition 2.4 A function $f \in \mathcal{C}\left(\mathbb{R}^{N} \times \Omega ; X\right)$ is called almost periodic in $x \in \mathbb{R}^{N}$ and uniformly on compact subsets of $\Omega$, if for every $\epsilon>0$ and compact $K \subset \Omega$, there exists a relatively dense set $T(f, \epsilon, K)$ in $\mathbb{R}^{N}$ such that

$$
\sup _{y \in K}\|f(\cdot+\tau, y)-f(\cdot, y)\|_{\infty} \leq \epsilon, \quad \forall \tau \in T(f, \epsilon, K) .
$$

Denote by $\mathcal{A P}\left(\mathbb{R}^{N} \times \Omega ; X\right)$ the set of all such functions.

Lemma 2.5 If $f \in \mathcal{A P}\left(\mathbb{R}^{N} \times \Omega ; X\right)$, then it is uniformly continuous on $\mathbb{R}^{N} \times K$ for each compact subset $K \subset \Omega$.

Proof For $\epsilon>0$, let $l$ be the length associated with the relatively dense set $T(f, \epsilon / 3, K)$ in $\mathbb{R}^{N}$ such that $T(f, \epsilon / 3, K) \cap B(x, l) \neq \emptyset$, for all $x \in \mathbb{R}^{N}$. Since $f$ is uniformly continuous on $B(0, l+1) \times K$, there is a $1>\delta>0$ such that when $\left(x^{\prime}, Z^{\prime}\right),\left(x^{\prime \prime}, Z^{\prime \prime}\right) \in B(0, l+1) \times K,\left|x^{\prime}-x^{\prime \prime}\right|$ and $\left|Z^{\prime}-Z^{\prime \prime}\right|<\delta$, one has

$$
\left\|f\left(x^{\prime}, Z^{\prime}\right)-f\left(x^{\prime \prime}, Z^{\prime \prime}\right)\right\|<\frac{\epsilon}{3} .
$$

Let $(s, Z),(t, W) \in \mathbb{R}^{N} \times K$ be such that

$$
|s-t|<\delta, \quad|Z-W|<\delta .
$$


Choosing an $\tau \in T(f, \epsilon / 3, K) \cap B(-s, l)$, then one has $s+\tau \in B(0, l)$ and $t+\tau \in B(0, l+1)$. Therefore, $(s+\tau, Z)$ and $(t+\tau, W) \in B(0, l+1) \times K$. It follows from inequalities (2.2) and (2.3) that

$$
\begin{aligned}
\|f(t, W)-f(s, Z)\| \leq & \|f(t, W)-f(t+\tau, W)\|+\|f(t+\tau, W)-f(s+\tau, Z)\| \\
& +\|f(s+\tau, Z)-f(s, Z)\|<\frac{\epsilon}{3}+\frac{\epsilon}{3}+\frac{\epsilon}{3}=\epsilon .
\end{aligned}
$$

The proof is completed.

\section{Pseudo almost periodic functions}

Denote by $\mathcal{P} \mathcal{A} \mathcal{P}_{0}\left(\mathbb{R}^{N} ; X\right)$ the set of ergodic perturbations $\varphi \in \mathcal{B C}\left(\mathbb{R}^{N} ; X\right)$ for which

$$
\lim _{r \rightarrow \infty} \frac{1}{m(B(0, r))} \int_{B(0, r)}\|\varphi(x)\| \mathrm{d} x=0 .
$$

It is easy to see that $\mathcal{P} \mathcal{A} \mathcal{P}_{0}\left(\mathbb{R}^{N} ; X\right)$ is a closed subspace of $\mathcal{B C}\left(\mathbb{R}^{N} ; X\right)$ and it contains the space of functions which vanish at infinite as a proper subspace.

As (2.1), (3.1) also has another version.

Lemma 3.1 Let $\varphi \in \mathcal{B C}\left(\mathbb{R}^{N} ; X\right)$. Then $\varphi \in \mathcal{P} \mathcal{A} \mathcal{P}_{0}\left(\mathbb{R}^{N} ; X\right)$ if and only if

$$
\lim _{r \rightarrow \infty} \frac{1}{m\left(K_{r}\right)} \int_{K_{r}}\|\varphi(x)\| \mathrm{d} x=0 .
$$

Proof According to the volume formulas of cubes and balls in $\mathbb{R}^{N}$, we have

$$
m(B(0, r))=c(N) m\left(K_{r / \sqrt{N}}\right)=C(N) m\left(K_{r}\right)
$$

where $c(N)$ and $C(N)$ are positive constants.

For each $r>0$, the inclusion $K_{r / \sqrt{N}} \subset B(0, r) \subset K_{r}$ holds in $\mathbb{R}^{N}$ with the usual Euclidean norm. It follows that

$$
\int_{K_{r / \sqrt{N}}}\|\varphi(x)\| \mathrm{d} x \leq \int_{B(0, r)}\|\varphi(x)\| \mathrm{d} x \leq \int_{K_{r}}\|\varphi(x)\| \mathrm{d} x .
$$

Dividing the inequalities by $m(B(0, r))$ yields

$$
\begin{aligned}
\frac{1}{c(N)} \frac{1}{m\left(K_{r / \sqrt{N}}\right)} \int_{K_{r / \sqrt{N}}}\|\varphi(x)\| \mathrm{d} x & \leq \frac{1}{m(B(0, r))} \int_{B(0, r)}\|\varphi(x)\| \mathrm{d} x \\
& \leq \frac{1}{C(N)} \frac{1}{m\left(K_{r}\right)} \int_{K_{r}}\|\varphi(x)\| \mathrm{d} x .
\end{aligned}
$$

Letting $r \rightarrow \infty$ in the formula above, we will get the equivalence of equations (3.1) and (3.2).

We use (3.1) or (3.2) in different occasions for convenience. For example, (3.1) will be used in Theorem 4.6 and (3.2) in Lemma 3.6. 
Definition 3.2 A closed subset $C$ of $\mathbb{R}^{N}$ is said to be an ergodic zero set in $\mathbb{R}^{N}$ if $m(C \cap$ $\left.K_{r}\right) / m\left(K_{r}\right) \rightarrow 0$ as $r \rightarrow \infty$.

Theorem 3.3 A function $\varphi \in \mathcal{B C}\left(\mathbb{R}^{N} ; X\right)$ is in $\mathcal{P} \mathcal{A} \mathcal{P}_{0}\left(\mathbb{R}^{N} ; X\right)$ if and only if for each $\epsilon>0$, the set $C_{\epsilon}=\left\{x \in \mathbb{R}^{N}:\|\varphi(x)\| \geq \epsilon\right\}$ is an ergodic zero set in $\mathbb{R}^{N}$.

Proof If $\varphi \in \mathcal{P} \mathcal{A} \mathcal{P}_{0}\left(\mathbb{R}^{N} ; X\right)$, we prove for each $\epsilon>0$ the set $C_{\epsilon}$ is an ergodic zero set in $\mathbb{R}^{N}$, i.e.,

$$
\lim _{r \rightarrow \infty} \frac{m\left(C_{\epsilon} \cap K_{r}\right)}{m\left(K_{r}\right)}=0
$$

Suppose to the contrary that there exists $\epsilon_{0}>0$ such that $m\left(C_{\epsilon_{0}} \cap K_{r}\right) / m\left(K_{r}\right)$ does not converge to 0 as $r \rightarrow \infty$. Then there exists $\delta>0$ such that for each $n$,

$$
\frac{m\left(C_{\epsilon_{0}} \cap K_{r_{n}}\right)}{m\left(K_{r_{n}}\right)} \geq \delta, \quad \text { for some } r_{n} \geq n .
$$

Thus, we have

$$
\frac{1}{m\left(K_{r_{n}}\right)} \int_{K_{r_{n}}}\|\varphi(x)\| \mathrm{d} x \geq \frac{1}{m\left(K_{r_{n}}\right)} \int_{K_{r_{n}} \cap C_{\epsilon_{0}}}\|\varphi(x)\| \mathrm{d} x \geq \epsilon_{0} \frac{m\left(C_{\epsilon_{0}} \cap K_{r_{n}}\right)}{m\left(K_{r_{n}}\right)} \geq \epsilon_{0} \delta,
$$

which contradicts the fact that $\varphi \in \mathcal{P} \mathcal{A} \mathcal{P}_{0}\left(\mathbb{R}^{N} ; X\right)$. So (3.3) holds.

On the other hand, suppose that (3.3) is true. That is, for any $\epsilon>0$, there exists $r_{0}>0$ such that for $r>r_{0}$,

$$
\frac{m\left(K_{r} \cap C_{\epsilon}\right)}{m\left(K_{r}\right)}<\frac{\epsilon}{\|\varphi\|_{\infty}+1} .
$$

Thus,

$$
\begin{aligned}
\frac{1}{m\left(K_{r}\right)} \int_{K_{r}}\|\varphi(x)\| \mathrm{d} x & =\frac{1}{m\left(K_{r}\right)} \int_{K_{r} \cap C_{\epsilon}}\|\varphi(x)\| \mathrm{d} x+\frac{1}{m\left(K_{r}\right)} \int_{K_{r} \backslash C_{\epsilon}}\|\varphi(x)\| \mathrm{d} x \\
& \leq \frac{m\left(K_{r} \cap C_{\epsilon}\right)}{m\left(K_{r}\right)}\|\varphi\|_{\infty}+\frac{1}{m\left(K_{r}\right)} \int_{K_{r} \backslash C_{\epsilon}} \epsilon \mathrm{d} x \\
& <\epsilon+\epsilon=2 \epsilon
\end{aligned}
$$

for $r>r_{0}$. This implies that $\varphi \in \mathcal{P} \mathcal{A} \mathcal{P}_{0}\left(\mathbb{R}^{N} ; X\right)$.

Lemma 3.4 The following statements hold:

(1) A function $\varphi \in \mathcal{B C}\left(\mathbb{R}^{N} ; E\right)$ is in $\mathcal{P} \mathcal{A} \mathcal{P}_{0}\left(\mathbb{R}^{N} ; E\right)$ if and only if $\varphi^{2}$ is.

(2) For each positive integer $n, \Phi \in \mathcal{B C}\left(\mathbb{R}^{N} ; E\right)^{n}$ is in $\mathcal{P} \mathcal{A} \mathcal{P}_{0}\left(\mathbb{R}^{N} ; E\right)^{n}$ if and only if the norm function $|\Phi(\cdot)|$ is in $\mathcal{P} \mathcal{A} \mathcal{P}_{0}\left(\mathbb{R}^{N} ; \mathbb{R}\right)$.

Proof (1) For any $\epsilon>0$, it is obvious that

$$
C_{\epsilon}(\varphi)=\left\{x \in \mathbb{R}^{N}:|\varphi(x)| \geq \epsilon\right\}=\left\{x \in \mathbb{R}^{N}:\left|\varphi(x)^{2}\right| \geq \epsilon^{2}\right\}=C_{\epsilon^{2}}\left(\varphi^{2}\right) .
$$

Since the $\epsilon>0$ is arbitrary, Theorem 3.3 implies the conclusion. 
(2) By (1), $\Phi=\left(\varphi_{1}, \varphi_{2}, \ldots, \varphi_{n}\right) \in \mathcal{P} \mathcal{A} \mathcal{P}_{0}\left(\mathbb{R}^{N} ; E\right)^{n}$ if and only if $\varphi_{i}^{2} \in \mathcal{P} \mathcal{A} \mathcal{P}_{0}\left(\mathbb{R}^{N} ; E\right), i=$ $1,2, \ldots, n$, the latter is equivalent to $|\Phi(\cdot)|^{2}=\sum_{i=1}^{n}\left|\varphi_{i}(\cdot)\right|^{2} \in \mathcal{P} \mathcal{A} \mathcal{P}_{0}\left(\mathbb{R}^{N} ; \mathbb{R}\right)$, which, again by (1), is equivalent to $|\Phi(\cdot)| \in \mathcal{P} \mathcal{A} \mathcal{P}_{0}\left(\mathbb{R}^{N} ; \mathbb{R}\right)$.

Definition 3.5 A function $f \in \mathcal{C}\left(\mathbb{R}^{N} ; X\right)$ is called pseudo almost periodic if $f=g+\varphi$, where $g \in \mathcal{A P}\left(\mathbb{R}^{N} ; X\right)$ and $\varphi \in \mathcal{P} \mathcal{A} \mathcal{P}_{0}\left(\mathbb{R}^{N} ; X\right)$. The functions $g$ and $\varphi$ are called the almost periodic component and ergodic perturbation of $f$, respectively. Denote by $\mathcal{P} \mathcal{A P}\left(\mathbb{R}^{N} ; X\right)$ the space of all such pseudo almost periodic functions.

The decomposition for $f$ above is unique. Otherwise, let $f=\tilde{g}+\tilde{\varphi}$ where $\tilde{g}$ is almost periodic and $\tilde{\varphi}$ is an ergodic perturbation. Then $g-\tilde{g}=\varphi-\tilde{\varphi}$. If $g-\tilde{g} \equiv 0$ does not hold, then by Lemma 2.3(2), $M(\|g-\tilde{g}\|)>0$. But since both $\varphi$ and $\tilde{\varphi}$ are ergodic perturbations, $M(\|\varphi-\tilde{\varphi}\|)=0$. This is a contradiction.

Lemma 3.6 If $f \in \mathcal{P} \mathcal{A P}\left(\mathbb{R}^{N} ; X\right)$ and $g$ is its almost periodic component, then $g\left(\mathbb{R}^{N}\right) \subset$ $\overline{f\left(\mathbb{R}^{N}\right)}$. Therefore $\|f\|_{\infty} \geq\|g\|_{\infty} \geq \inf _{x \in \mathbb{R}^{N}}\|g(x)\| \geq \inf _{x \in \mathbb{R}^{N}}\|f(x)\|$.

Proof If $g\left(\mathbb{R}^{N}\right) \nsubseteq \overline{f\left(\mathbb{R}^{N}\right)}$, then there exists an $x_{0} \in \mathbb{R}^{N}$ such that $\inf _{s \in \mathbb{R}^{N}}\left\|g\left(x_{0}\right)-f(s)\right\|>$ $\epsilon>0$. Since $g$ is continuous at $x_{0}$, there is a $\delta>0$ such that $|x|<\delta$ implies that inf $\operatorname{in}_{s \in \mathbb{R}^{N}} \| g\left(x_{0}+\right.$ $x)-f\left(x_{0}+s\right) \|>\epsilon$. By Definition 2.1, for $\epsilon>0$ there exists $l_{\epsilon / 2}>0$ such that every cube $-x_{0}+s+K_{l_{\epsilon / 2}}, s \in \mathbb{R}^{N}$ contains at least one point $\tau \in T(g, \epsilon / 2)$. Therefore, if $|x|<\delta$, then

$$
\begin{aligned}
\left\|\varphi\left(x_{0}+x+\tau\right)\right\| & =\left\|f\left(x_{0}+x+\tau\right)-g\left(x_{0}+x+\tau\right)\right\| \\
& \geq\left\|f\left(x_{0}+x+\tau\right)-g\left(x_{0}+x\right)\right\|-\left\|g\left(x_{0}+x\right)-g\left(x_{0}+x+\tau\right)\right\| \\
& \geq \inf _{s \in \mathbb{R}^{N}}\left\|f\left(x_{0}+s\right)-g\left(x_{0}+x\right)\right\|-\left\|g\left(x_{0}+\cdot\right)-g\left(x_{0}+\cdot+\tau\right)\right\|_{\infty} \\
& >\in / 2 .
\end{aligned}
$$

This implies that each cube $s+K_{l_{\epsilon / 2}+\delta}, s \in \mathbb{R}^{N}$ contains a ball with radius $\delta$ on which $\|\varphi\|>$ $\epsilon / 2$.

Now, we use (3.2) to show the contradiction. For any positive integer $n$, we divide $K_{n\left(l_{\epsilon / 2}+\delta\right)}$ into $n^{N}$ smaller cubes with edge length $l_{\epsilon / 2}+\delta$ who do not have common inner point with each other. Then, using the volume formulas $m\left(K_{r}\right)=(2 r)^{N}$ and $m(B(0, r))=$ $\left[\pi^{N / 2} / \Gamma(1+N / 2)\right] \cdot r^{N}$ in $\mathbb{R}^{N}$ where $\Gamma(\cdot)$ is the classical Gamma function, we obtain

$$
\begin{aligned}
\frac{1}{m\left(K_{n\left(l_{\epsilon / 2}+\delta\right)}\right)} \int_{K_{n\left(l_{\epsilon / 2}+\delta\right)}\|\varphi(x)\| \mathrm{d} x} \geq \frac{1}{m\left(K_{n\left(l_{\epsilon / 2}+\delta\right)}\right)} \cdot n^{N} \cdot m(B(0, \delta)) \cdot \frac{\epsilon}{2} \\
=\frac{n^{N}}{\left(2 n\left(l_{\epsilon / 2}+\delta\right)\right)^{N}} \cdot\left[\pi^{N / 2} / \Gamma(1+N / 2)\right] \cdot \delta^{N} \cdot \frac{\epsilon}{2} \\
=\frac{\left[\pi^{N / 2} / \Gamma(1+N / 2)\right] \cdot \delta^{N}}{2^{N}\left(l_{\epsilon / 2}+\delta\right)^{N}} \frac{\epsilon}{2}>0 .
\end{aligned}
$$

Note that the last term in the formula above is independent of $n$. Letting $n \rightarrow \infty$ above, because $\varphi \in \mathcal{P} \mathcal{A} \mathcal{P}_{0}\left(\mathbb{R}^{N} ; X\right)$, we get a contradiction to (3.2).

Theorem 3.7 $\mathcal{P} \mathcal{A} \mathcal{P}\left(\mathbb{R}^{N} ; X\right)$ is a Banach space. 
Proof Obviously $\mathcal{P} \mathcal{A} \mathcal{P}\left(\mathbb{R}^{N} ; X\right)$ is a normed linear subspace of $\mathcal{B C}\left(\mathbb{R}^{N} ; X\right)$, and we only need to show it is complete. Let $\left\{f_{n}\right\} \subset \mathcal{P} \mathcal{A} \mathcal{P}\left(\mathbb{R}^{N} ; X\right)$ be a Cauchy sequence, $g_{n}$ and $\varphi_{n}$ be the almost periodic component and ergodic perturbation of $f_{n}$, respectively. By Lemma 3.6, $\left\{g_{n}\right\} \subset \mathcal{A P}\left(\mathbb{R}^{N} ; X\right)$ is Cauchy too. So is $\left\{\varphi_{n}\right\} \subset \mathcal{P} \mathcal{A} \mathcal{P}_{0}\left(\mathbb{R}^{N} ; X\right)$. Since $\mathcal{A P}\left(\mathbb{R}^{N} ; X\right)$ and $\mathcal{P} \mathcal{A} \mathcal{P}_{0}\left(\mathbb{R}^{N} ; X\right)$ are closed in $\mathcal{B C}\left(\mathbb{R}^{N} ; X\right)$, there exist $g \in \mathcal{A P}\left(\mathbb{R}^{N} ; X\right)$ and $\varphi \in \mathcal{P} \mathcal{A} \mathcal{P}_{0}\left(\mathbb{R}^{N} ; X\right)$ such that $\left\|g_{n}-g\right\|_{\infty} \rightarrow 0$ and $\left\|\varphi_{n}-\varphi\right\|_{\infty} \rightarrow 0$ as $n \rightarrow \infty$. Set $f=g+\varphi$. Then $f \in$ $\mathcal{P} \mathcal{A} \mathcal{P}\left(\mathbb{R}^{N} ; X\right)$ and $\left\|f_{n}-f\right\|_{\infty} \rightarrow 0$, as $n \rightarrow \infty$.

Definition 3.8 A function $f \in \mathcal{C}\left(\mathbb{R}^{N} \times \Omega ; X\right)$ is called an ergodic perturbation in $x \in \mathbb{R}^{N}$ and uniformly on compact subsets of $\Omega$, if for each compact $K \subset \Omega, f \in \mathcal{B C}\left(\mathbb{R}^{N} \times K ; X\right)$ and

$$
\lim _{r \rightarrow \infty} \frac{1}{m(B(0, r))} \int_{B(0, r)}\|\varphi(x, Z)\| \mathrm{d} x=0
$$

uniformly with respect to $Z \in K$.

Let $\mathcal{P} \mathcal{A} \mathcal{P}\left(\mathbb{R}^{N} \times \Omega ; X\right)$ denote all the functions $f$ of the form

$$
f=g+\varphi,
$$

where $g \in \mathcal{A} \mathcal{P}\left(\mathbb{R}^{N} \times \Omega ; X\right)$ and $\varphi \in \mathcal{P} \mathcal{A} \mathcal{P}_{0}\left(\mathbb{R}^{N} \times \Omega ; X\right)$.

Let $K$ be a subset of $\Omega$. A function $f: \mathbb{R}^{N} \times \Omega \rightarrow X$ is said to be continuous in $z \in K$ and uniformly in $x \in \mathbb{R}^{N}$, if for any given $z \in K$ and $\epsilon>0$, there exists a $\delta(z, \epsilon)>0$, such that $z^{\prime} \in K$ and $\left|z^{\prime}-z\right|<\delta(z, \epsilon)$ imply that $\left\|f\left(x, z^{\prime}\right)-f(x, z)\right\|<\epsilon$ for all $x \in \mathbb{R}^{N}$.

Lemma 3.9 Suppose the function $f \in \mathcal{P} \mathcal{A} \mathcal{P}_{0}\left(\mathbb{R}^{N} \times \Omega ; X\right)$ is continuous in $z \in K$ and uniformly in $x \in \mathbb{R}^{N}$ for a compact $K \subset \Omega$. Then

$$
\lim _{r \rightarrow \infty} \frac{1}{m(B(0, r))} \int_{B(0, r)} \max _{z \in K}\|f(x, z)\| \mathrm{d} x=0 .
$$

Proof Since $K$ is compact, for arbitrary $\epsilon>0$ one can find a finite number, say $m$, of open balls $B_{k}$ with center $z^{(k)} \in K$ and radius $\delta\left(z^{(k)}, \frac{\epsilon}{2}\right), k=1,2, \ldots, m$, such that $K \subset \bigcup_{k=1}^{m} B_{k}$ and

$$
\left\|f(x, z)-f\left(x, z^{(k)}\right)\right\|<\frac{\epsilon}{2} \quad\left(z \in B_{k}, x \in \mathbb{R}^{N}\right)
$$

Since each $f\left(\cdot, z^{(k)}\right) \in \mathcal{P} \mathcal{A} \mathcal{P}_{0}\left(\mathbb{R}^{N} ; X\right)$, there is a number $r_{0}>0$ such that

$$
\sum_{k=1}^{m} \frac{1}{m(B(0, r))} \int_{B(0, r)}\left\|f\left(x, z^{(k)}\right)\right\| \mathrm{d} x<\frac{\epsilon}{2}, \quad \forall r>r_{0} .
$$

It follows from (3.4) and (3.5) that

$$
\frac{1}{m(B(0, r))} \int_{B(0, r)} \max _{z \in K}\|f(x, z)\| \mathrm{d} x \leq \frac{1}{m(B(0, r))} \int_{B(0, r)} \sum_{k=1}^{m}\left\|f\left(x, z^{(k)}\right)\right\| \mathrm{d} x+\frac{\epsilon}{2}<\epsilon
$$

for $r>r_{0}$. By the arbitrariness of $\epsilon$, we get the conclusion. 


\section{Almost periodic and pseudo almost periodic weak solutions}

In this section, we simplify the notations for $\mathbb{R}$-valued function spaces in the previous sections by omitting the value space. For example, $\mathcal{C}\left(\mathbb{R}^{N} ; \mathbb{R}\right)$ is replaced with $\mathcal{C}\left(\mathbb{R}^{N}\right)$. Also some new notations will be used in this section, i.e., $\mathcal{C}^{1}\left(\mathbb{R}^{N}\right)$ is the set of all continuous differentiable functions; $\mathcal{C}_{0}^{\infty}\left(\mathbb{R}^{N}\right)$ denotes the set all smooth functions with compact support; $H_{\text {loc }}^{1}\left(\mathbb{R}^{N}\right)$ denotes the local Sobolev space of functions which and their first generalized derivatives are locally square integrable; and $L^{\infty}\left(\mathbb{R}^{N}\right)\left(L_{\text {loc }}^{\infty}\left(\mathbb{R}^{N}\right)\right)$ is the Lebesgue measurable (locally) bounded function space.

Now, we consider the problem

$$
\left\{\begin{array}{l}
-\Delta u+\sum_{j=1}^{N} c_{j} \partial_{j} u+f(x, u)=h(x), \quad x \in \mathbb{R}^{N}, \\
u \in \mathcal{C}\left(\mathbb{R}^{N}\right)
\end{array}\right.
$$

where $c_{j}$ 's are real constants, $f \in \mathcal{C}\left(\mathbb{R}^{N} \times \mathbb{R}\right)$, and $h \in L_{\text {loc }}^{\infty}\left(\mathbb{R}^{N}\right)$.

Definition 4.1 The function $\alpha \in H_{\mathrm{loc}}^{1}\left(\mathbb{R}^{N}\right) \cap L_{\mathrm{loc}}^{\infty}\left(\mathbb{R}^{N}\right)$ is a lower solution of (4.1) if, for every $v \in \mathcal{C}_{0}^{\infty}\left(\mathbb{R}^{N}\right)$,

$$
v \geq 0 \Rightarrow \int_{\mathbb{R}^{N}} \sum_{j=1}^{N} \partial_{j} \alpha\left(\partial_{j} v+c_{j} v\right) \mathrm{d} x+\int_{\mathbb{R}^{N}} f(x, \alpha) v \mathrm{~d} x-\int_{\mathbb{R}^{N}} h v \mathrm{~d} x \leq 0 .
$$

The function $\beta \in H_{\text {loc }}^{1}\left(\mathbb{R}^{N}\right) \cap L_{\text {loc }}^{\infty}\left(\mathbb{R}^{N}\right)$ is an upper solution of (4.1) if, for every $v \in \mathcal{C}_{0}^{\infty}\left(\mathbb{R}^{N}\right)$,

$$
v \geq 0 \Rightarrow \int_{\mathbb{R}^{N}} \sum_{j=1}^{N} \partial_{j} \beta\left(\partial_{j} v+c_{j} v\right) \mathrm{d} x+\int_{\mathbb{R}^{N}} f(x, \beta) v \mathrm{~d} x-\int_{\mathbb{R}^{N}} h v \mathrm{~d} x \geq 0 .
$$

For the existence of a weak solution to (4.1) and its relationship with the nonlinear term $f$ and forcing term $h$, we refer to the following two lemmas.

Lemma 4.2 ([14, Theorem 2.5]) Assume that $\alpha$ is a lower and $\beta$ an upper solution of (4.1) and $\alpha \leq \beta$. Then problem (4.1) has a weak solution $u \in \mathcal{C}^{1}\left(\mathbb{R}^{N}\right)$ such that $\alpha \leq u \leq \beta$.

Lemma 4.3 ([14, Theorem 2.7]) Let $h_{1}, h_{2}, \alpha, \beta \in L^{\infty}\left(\mathbb{R}^{N}\right)$. Assume that there exists $\delta>0$ such that

$$
\alpha(x) \leq s \leq t \leq \beta(x) \quad \Rightarrow \quad f(x, t)-f(x, s) \geq \delta(t-s) .
$$

If, for $i=1,2, u_{i} \in \mathcal{C}^{1}\left(\mathbb{R}^{N}\right)$ is a weak solution of

$$
-\Delta u+\sum_{j=1}^{N} c_{j} \partial_{j} u+f(x, u)=h_{i}(x)
$$

such that $\alpha \leq u_{i} \leq \beta$, then

$$
\left\|u_{1}-u_{2}\right\| \leq \delta^{-1}\left\|h_{1}-h_{2}\right\| .
$$


In the remaining of this section, we will consider the almost periodicity and pseudo almost periodicity of the bounded weak solution of problem (4.1) under the assumption that $f$ and $h$ have the corresponding properties.

\subsection{Almost periodic weak solution}

To show the pseudo almost periodicity of a weak solution $u$ of problem (4.1), the process consists of two parts: obtaining its almost periodic component and then the ergodic perturbation. This subsection is devoted to obtaining the almost periodic component.

Theorem 4.4 Under the assumptions of Lemma 4.2, we assume further that $\alpha, \beta \in$ $L^{\infty}\left(\mathbb{R}^{N}\right)$ and there exists $\delta>0$ such that

$$
\operatorname{essinf} \alpha \leq s \leq t \leq \operatorname{ess} \sup \beta \quad \Rightarrow \quad f(x, t)-f(x, s) \geq \delta(t-s)
$$

where ess inf (ess sup) denotes the essential infimum (essential supremum) of a function. We also assume that $f$ is almost periodic in $x \in \mathbb{R}^{N}$ and uniformly on compact subsets of $\mathbb{R}$, and $h$ is almost periodic. Then the weak solution $u$ of (4.1) in Lemma 4.2 is unique and almost periodic.

Proof The uniqueness of $u$ follows from Lemma 4.3.

From the assumptions, we know that $f$ is almost periodic in $x \in \mathbb{R}^{N}$ and uniformly on the compact set [ess inf $\alpha$, ess $\sup \beta] \subset \mathbb{R}$. By [12, Theorem 9.4], there exist a Banach space $Y$ and an almost periodic function $\vec{f}: \mathbb{R}^{N} \rightarrow Y$ such that

$$
\|f(\cdot+\tau, u)-f(\cdot, u)\|_{\infty} \leq\|\vec{f}(\cdot+\tau)-\vec{f}(\cdot)\|_{\infty}, \quad \forall \tau \in \mathbb{R}^{N}, \forall u \in[\operatorname{essinf} \alpha, \operatorname{ess} \sup \beta] .
$$

For an arbitrary $\epsilon>0$, it follows from Lemma 2.2(5) that $T\left(h, \delta \frac{\epsilon}{2}\right) \cap T\left(\vec{f}, \delta \frac{\epsilon}{2}\right)$ is relatively dense in $\mathbb{R}^{N}$. We will show that $T\left(h, \delta \frac{\epsilon}{2}\right) \cap T\left(\vec{f}, \delta \frac{\epsilon}{2}\right) \subset T(u, \epsilon)$, and thus $u$ is almost periodic on $\mathbb{R}^{N}$.

Let $\tau \in T\left(h, \delta \frac{\epsilon}{2}\right) \cap T\left(\vec{f}, \delta \frac{\epsilon}{2}\right)$. Then $u(\cdot+\tau)$ is a weak solution of the equation

$$
\begin{aligned}
&-\Delta u(x+\tau)+\sum_{j=1}^{N} c_{j} \partial_{j} u(x+\tau)+f(x, u(x+\tau)) \\
&=h(x+\tau)+f(x, u(x+\tau))-f(x+\tau, u(x+\tau)) .
\end{aligned}
$$

Note that both $u$ and $u(\cdot+\tau)$ satisfy the condition in (4.3). We can take the constants ess inf $\alpha$ and ess $\sup \beta$ as two functions in $L^{\infty}\left(\mathbb{R}^{N}\right)$, then Lemma 4.3 implies that

$$
\begin{aligned}
\|u-u(\cdot+\tau)\|_{\infty} & \leq \delta^{-1}\|h(\cdot+\tau)-h(\cdot)+f(\cdot, u(\cdot+\tau))-f(\cdot+\tau, u(\cdot+\tau))\|_{\infty} \\
& \leq \delta^{-1}\left(\|h(\cdot+\tau)-h(\cdot)\|_{\infty}+\|\vec{f}(\cdot)-\vec{f}(\cdot+\tau)\|_{\infty}\right) \\
& \leq \epsilon
\end{aligned}
$$

i.e., $\tau \in T(u, \epsilon)$. The proof is completed. 
Remark 4.5 Theorem 4.4 improves [14, Theorem 3.2] where the nonlinear term $f(x, u) \equiv$ $f(u)$, which benefits from the applications of the notion of almost periodic dominance $\vec{f}$ (see [12, Chapter 9]) and Lemma 2.2(5).

\subsection{Pseudo almost periodic weak solution}

Now we assume that $f$ is pseudo almost periodic in $x \in \mathbb{R}^{N}$ and uniformly on compact subsets of $\mathbb{R}$ and $h$ is also pseudo almost periodic. Let

$$
f(x, u)=g(x, u)+\varphi(x, u), \quad h(x)=h_{1}(x)+h_{2}(x), \quad \forall x \in \mathbb{R}^{N}, \forall u \in \mathbb{R}
$$

where $g$ and $h_{1}$ are the almost periodic components, $\varphi$ and $h_{2}$ are ergodic perturbations.

In the following, we shall use the auxiliary nonnegative function $\psi_{R} \in \mathcal{C}_{0}^{\infty}\left(\mathbb{R}^{N}\right), R \geq 1$, such that for some $\kappa \in(0,1)$,

$$
\begin{aligned}
& \psi_{R}(x)= \begin{cases}1, & |x| \leq R, \\
0, & |x| \geq R+R^{\kappa},\end{cases} \\
& \left|\partial^{\gamma} \psi_{R}(x)\right| \leq C_{\gamma} \cdot R^{-\kappa|\gamma|},
\end{aligned}
$$

where $\gamma=\left(\gamma_{1}, \gamma_{2}, \ldots, \gamma_{n}\right)$ is the multiple index with the $\gamma_{i}^{\prime}$ 's being nonnegative integers, $|\gamma|=\sum_{i=1}^{N} \gamma_{i}, \partial^{\gamma}=\frac{\partial^{|\gamma|}}{\partial x_{1}^{1 / \ldots \partial x_{N}^{\gamma N}}}, C_{\gamma}>0$ does not depend on $R$. The function $\psi_{R}$ originates from [17, Lemma 4.1] and has been used in [13, page 159] for another purpose.

To consider (4.1), we also need to consider its almost periodic component, i.e. the equation

$$
\left\{\begin{array}{l}
-\Delta v+\sum_{j=1}^{N} c_{j} \partial_{j} v+g(x, v)=h_{1}(x), \quad x \in \mathbb{R}^{N}, \\
v \in \mathcal{C}\left(\mathbb{R}^{N}\right) .
\end{array}\right.
$$

Theorem 4.6 Assume that $\alpha_{1}, \alpha_{2} \in H_{\mathrm{loc}}^{1}\left(\mathbb{R}^{N}\right) \cap L^{\infty}\left(\mathbb{R}^{N}\right)$ are lower and $\beta_{1}, \beta_{2} \in H_{\mathrm{loc}}^{1}\left(\mathbb{R}^{N}\right) \cap$ $L^{\infty}\left(\mathbb{R}^{N}\right)$ upper solutions of (4.1) and (4.4), respectively. Let $f$ be continuous in $z \in K$ and uniformly in $x \in \mathbb{R}^{N}$ for each compact $K \subset \mathbb{R}$. If there exists $\delta>0$ such that

$$
\begin{aligned}
& \text { essinf } \min \left\{\alpha_{1}, \alpha_{2}\right\} \leq s \leq t \leq \operatorname{ess} \sup \max \left\{\beta_{1}, \beta_{2}\right\} \\
& \Rightarrow f(x, t)-f(x, s) \geq \delta(t-s)
\end{aligned}
$$

then problem (4.1) has a unique pseudo almost periodic weak solution $u \in \mathcal{C}^{1}\left(\mathbb{R}^{N}\right)$ such that $\alpha_{1} \leq u \leq \beta_{1}$ whose almost periodic component is a weak solution of (4.4).

Proof It follows from Lemma 3.6 that (4.5) is also valid when $f$ is replaced with $g$. In fact, for each pair of $s$ and $t$ satisfying the condition in (4.5), $g(\cdot, t)-g(\cdot, s)$ is the almost periodic component of the pseudo almost periodic function $f(\cdot, t)-f(\cdot, s)$. By Lemma 3.6, for each $x \in \mathbb{R}^{N}$ there exists a sequence $\left\{x_{n}\right\} \subset \mathbb{R}^{N}$ such that

$$
g(x, t)-g(x, s)=\lim _{n \rightarrow \infty} f\left(x_{n}, t\right)-f\left(x_{n}, s\right) \geq \delta(t-s) .
$$


Lemmas 4.2 and 4.3 imply the existence and uniqueness of weak solutions $u \in \mathcal{C}^{1}\left(\mathbb{R}^{N}\right)$ and $v \in \mathcal{C}^{1}\left(\mathbb{R}^{N}\right)$ of (4.1) and (4.4), respectively, such that $\alpha_{1} \leq u \leq \beta_{1}$ and $\alpha_{2} \leq v \leq \beta_{2}$. Moreover, Theorem 4.4 implies that $v$ is almost periodic.

Now if we can show that $u-v \in \mathcal{P} \mathcal{A} \mathcal{P}_{0}\left(\mathbb{R}^{N}\right)$, then the proof is completed.

Let $w=u-v$, then $w$ is a weak solution of the equation

$$
-\Delta w+\sum_{j=1}^{N} c_{j} \partial_{j} w+f(x, u)-f(x, v)=h_{2}(x)-\varphi(x, v), \quad x \in \mathbb{R}^{N} .
$$

Since $w \in \mathcal{C}^{1}\left(\mathbb{R}^{N}\right)$ and $\psi_{R} \in \mathcal{C}_{0}^{\infty}\left(\mathbb{R}^{N}\right), w \cdot \psi_{R} \in H_{0}^{1}\left(\mathbb{R}^{N}\right)$. Thus, by the definition of a weak solution, we have

$$
\begin{aligned}
& \int_{B\left(0, R+R^{\kappa}\right)} \nabla w \cdot \nabla\left(w \cdot \psi_{R}\right) \mathrm{d} x+\int_{B\left(0, R+R^{\kappa}\right)} \sum_{j=1}^{N} c_{j} \partial_{j} w \cdot\left(w \cdot \psi_{R}\right) \mathrm{d} x \\
& \quad+\int_{B\left(0, R+R^{\kappa}\right)}[f(x, u)-f(x, v)]\left(w \cdot \psi_{R}\right) \mathrm{d} x \\
& =\int_{B\left(0, R+R^{\kappa}\right)}\left[h_{2}(x)-\varphi(x, v)\right]\left(w \cdot \psi_{R}\right) \mathrm{d} x .
\end{aligned}
$$

Now we will give some estimations for terms on the left side of (4.6). For the first term, we have

$$
\begin{aligned}
I_{1} & =\int_{B\left(0, R+R^{\kappa}\right)} \nabla w \cdot \nabla\left(w \cdot \psi_{R}\right) \mathrm{d} x \\
& =\int_{B\left(0, R+R^{\kappa}\right)} \nabla w \cdot \nabla w \cdot \psi_{R} \mathrm{~d} x+\int_{B\left(0, R+R^{\kappa}\right)} \nabla w \cdot w \cdot \nabla \psi_{R} \mathrm{~d} x .
\end{aligned}
$$

Since $\psi_{R}$ is nonnegative, $\int_{B\left(0, R+R^{k}\right)} \nabla w \cdot \nabla w \cdot \psi_{R} \mathrm{~d} x \geq 0$. Using integration by parts, it is easy to see that

$$
\begin{aligned}
& \int_{B\left(0, R+R^{\kappa}\right)} \nabla w \cdot w \cdot \nabla \psi_{R} \mathrm{~d} x \\
&=\sum_{j=1}^{N} \int_{B\left(0, R+R^{\kappa}\right)} \partial_{j}\left(\frac{1}{2} w^{2}\right) \cdot \partial_{j} \psi_{R} \mathrm{~d} x \\
& \geq-\sum_{j=1}^{N}\left[\int_{\left|x-x_{j} e_{j}\right| \leq R+R^{\kappa}} \mathrm{d} x_{1} \cdots \widehat{\mathrm{d} x_{j}} \cdots \mathrm{d} x_{N}\left\|w^{2} \cdot \partial_{j} \psi_{R}\right\|_{\infty}\right. \\
&\left.\quad+\int_{B\left(0, R+R^{\kappa}\right)} \frac{1}{2} w^{2} \cdot \partial_{j}^{2} \psi_{R} \mathrm{~d} x\right] \\
& \geq--N \cdot\left[\pi^{(N-1) / 2} / \Gamma(1+(N-1) / 2)\right]\left(R+R^{\kappa}\right)^{N-1} \cdot\|w\|_{\infty}^{2} \cdot \max _{|\gamma|=1}\left\{C_{\gamma}\right\} R^{-\kappa} \\
&-\frac{1}{2} N \cdot\left[\pi^{N / 2} / \Gamma(1+N / 2)\right]\left[\left(R+R^{\kappa}\right)^{N}-R^{N}\right] \cdot\|w\|_{\infty}^{2} \cdot \max _{|\gamma|=2}\left\{C_{\gamma}\right\} R^{-2 \kappa} \\
&=-\mathcal{O}\left(R^{N-1-\kappa}\right) \text { as } R \rightarrow \infty,
\end{aligned}
$$


where $e_{j} \in \mathbb{R}^{N}$ whose $j$ th element is 1 and the others are $0, \mathcal{O}$ is the symbol of infinite of the same order.

For the second term in (4.6), similar to the last formulas, we have

$$
\begin{aligned}
I_{2}= & \int_{B\left(0, R+R^{\kappa}\right)} \sum_{j=1}^{N} c_{j} \partial_{j} w \cdot\left(w \cdot \psi_{R}\right) \mathrm{d} x=\sum_{j=1}^{N} c_{j} \int_{B\left(0, R+R^{\kappa}\right)} \partial_{j}\left(\frac{1}{2} w^{2}\right) \cdot \psi_{R} \mathrm{~d} x \\
\geq & -\sum_{j=1}^{N}\left|c_{j}\right| \cdot\left[\pi^{(N-1) / 2} / \Gamma(1+(N-1) / 2)\right]\left(R+R^{\kappa}\right)^{N-1} \cdot\|w\|_{\infty}^{2} \cdot C_{\theta} \\
& -\frac{1}{2} \sum_{j=1}^{N}\left|c_{j}\right| \cdot\left[\pi^{(N-1) / 2} / \Gamma(1+(N-1) / 2)\right]\left[\left(R+R^{\kappa}\right)^{N}-R^{N}\right] \cdot\|w\|_{\infty}^{2} \max _{|\gamma|=1}\left\{C_{\gamma}\right\} R^{-\kappa} \\
= & -\mathcal{O}\left(R^{N-1}\right) \quad \text { as } R \rightarrow \infty,
\end{aligned}
$$

where $\theta=(0, \ldots, 0)$ is the zero index.

For the third term, it is implied by (4.5) that

$$
I_{3}=\int_{B\left(0, R+R^{\kappa}\right)}[f(x, u)-f(x, v)]\left(w \cdot \psi_{R}\right) \mathrm{d} x \geq \delta \int_{B(0, R)} w^{2} \mathrm{~d} x .
$$

Multiplying (4.6) by $\frac{1}{m\left(B\left(0, R+R^{K}\right)\right)}$ and using the estimations for $I_{1}-I_{3}$, we obtain for sufficiently large $R$,

$$
\begin{aligned}
& \delta \frac{1}{m\left(B\left(0, R+R^{\kappa}\right)\right)} \int_{B(0, R)} w^{2} \mathrm{~d} x \\
& \quad \leq C \cdot R^{-1}+C_{\theta}\|w\|_{\infty} \cdot \frac{1}{m\left(B\left(0, R+R^{\kappa}\right)\right)} \int_{B\left(0, R+R^{\kappa}\right)}\left(\left|h_{2}(x)\right|+|\varphi(x, v)|\right) \mathrm{d} x,
\end{aligned}
$$

where $C$ is a positive constant.

Since $h_{2} \in \mathcal{P} \mathcal{A} \mathcal{P}_{0}\left(\mathbb{R}^{N}\right)$, we have

$$
\lim _{R \rightarrow \infty} \frac{1}{m\left(B\left(0, R+R^{\kappa}\right)\right)} \int_{B\left(0, R+R^{\kappa}\right)}\left|h_{2}(x)\right| \mathrm{d} x=0
$$

By Lemma 2.2(2), the range $\mathcal{R}_{v}$ of $v$ is relatively compact. We know from Lemma 2.5 that $g(x, z)$ is uniformly continuous in $\mathbb{R}^{N} \times \overline{\mathcal{R}_{v}}$. Since $f(x, z)$ is continuous in $z \in \overline{\mathcal{R}_{v}}$ and uniformly in $x \in \mathbb{R}^{N}$, then so is $\varphi=f-g$. Now by Lemma 3.9 , we have

$$
\begin{aligned}
& \lim _{R \rightarrow \infty} \frac{1}{m\left(B\left(0, R+R^{\kappa}\right)\right)} \int_{B\left(0, R+R^{\kappa}\right)}|\varphi(x, v)| \mathrm{d} x \\
& \quad \leq \lim _{R \rightarrow \infty} \frac{1}{m\left(B\left(0, R+R^{\kappa}\right)\right)} \int_{B\left(0, R+R^{\kappa}\right)} \max _{z \in \mathcal{R}_{v}}|\varphi(x, z)| \mathrm{d} x=0 .
\end{aligned}
$$

It follows from (4.8)-(4.10) that

$$
\lim _{R \rightarrow \infty} \frac{1}{m\left(B\left(0, R+R^{\kappa}\right)\right)} \int_{B(0, R)} w^{2} \mathrm{~d} x=0 .
$$


Note that $\frac{m(B(0, R))}{m\left(B\left(0, R+R^{K}\right)\right)}=\frac{R^{N}}{\left(R+R^{K}\right)^{N}}=\frac{1}{\left(1+R^{\kappa-1}\right)} \rightarrow 1$, as $R \rightarrow \infty$. Then (4.11) implies that

$$
\lim _{R \rightarrow \infty} \frac{1}{m(B(0, R))} \int_{B(0, R)} w^{2} \mathrm{~d} x=0
$$

i.e., $w^{2} \in \mathcal{P} \mathcal{A} \mathcal{P}_{0}\left(\mathbb{R}^{N}\right)$. From Lemma 3.4, it follows that $w \in \mathcal{P} \mathcal{A} \mathcal{P}_{0}\left(\mathbb{R}^{N}\right)$.

Remark 4.7 Theorem 4.6 can also be comprehended in the following way. If the nonlinear term $g$ and forcing term $h_{1}$ of (4.4) are perturbed by ergodic perturbations which maintain the monotonicity of nonlinear term and the existence of upper and lower solutions, then its almost periodic weak solution is also perturbed by an ergodic perturbation.

\subsection{Pseudo almost periodic forced pendulum equation}

Consider the equation

$$
-\Delta u+\sum_{j=1}^{N} c_{j} \partial_{j} u-f(x) \cdot \sin u=h(x), \quad x \in \mathbb{R}^{N},
$$

where $c_{j}$ 's are real constants, $f$ and $h$ both are pseudo almost periodic. If there exists an $\epsilon>0$ such that $f(x) \geq\|h\|_{\infty}+\epsilon, \forall x \in \mathbb{R}^{N}$, then (4.12) has a unique pseudo almost periodic weak solution such that $\frac{\pi}{2}<u<\frac{3 \pi}{2}$.

A special case of (4.12) was treated in [14, Example 3.3] and [15, Example 7.1(b)], where $f(x) \equiv a, h$ is almost periodic and $a>\|h\|_{\infty}$.

Proof Let $g$ and $h_{1}$ are the almost periodic components of $f$ and $h$, respectively. We need to consider the following almost periodic equation together:

$$
-\Delta v+\sum_{j=1}^{N} c_{j} \partial_{j} v-g(x) \cdot \sin v=h_{1}(x), \quad x \in \mathbb{R}^{N} .
$$

It follows from Lemma 3.6 that $g$ and $h_{1}$ also satisfy the relationship that $g(x) \geq\left\|h_{1}\right\|_{\infty}+\epsilon$, $\forall x \in \mathbb{R}^{N}$.

If $\delta>0$ is sufficient small $(<\epsilon)$, then $\alpha=\frac{\pi}{2}+\delta$ and $\beta=\frac{3 \pi}{2}-\delta$ are lower and upper solutions of both (4.12) and (4.13).

Since $-\partial_{u}[f(x) \cdot \sin u]=-f(x) \cdot \cos u$ is strictly positive when $u \in\left[\frac{\pi}{2}+\delta, \frac{3 \pi}{2}-\delta\right]$.

Now we can apply Theorem 4.6 and get the conclusion that (4.12) has a unique pseudo almost periodic weak solution $u$ such that $\frac{\pi}{2}<u<\frac{3 \pi}{2}$ and its almost periodic component is a weak solution of (4.13).

Competing interests

The authors declare that they have no competing interests.

Authors' contributions

All authors contributed equally in this article. They read and approved the final manuscript.

Author details

'Department of Mathematics, Harbin Institute of Technology, West Dazhi Street, Harbin, 150001, China. ${ }^{2}$ Department of Mathematics, Harbin Institute of Technology, West Dazhi Street, Harbin, 150001, China. 


\section{Acknowledgements}

The authors acknowledge support from the NSF of China (no. 11071048)

Received: 11 December 2013 Accepted: 8 January 2014 Published: 29 Jan 2014

\section{References}

1. Zhang, CY: Integration of vector-valued pseudo almost periodic functions. Proc. Am. Math. Soc. 121, 167-174 (1994)

2. Zhang, CY: Pseudo almost periodic solutions of some differential equations. J. Math. Anal. Appl. 151, 62-76 (1994)

3. Zhang, CY: Pseudo almost periodic solutions of some differential equations, II. J. Math. Anal. Appl. 192, 543-561 (1995)

4. Li, HX, Huang, FL, Li, JY: Composition of pseudo almost-periodic functions and semilinear differential equations. J. Math. Anal. Appl. 255, 436-446 (2001)

5. Cieutat, P, Fatajou, S, N'Guérékata, GM: Composition of pseudo almost periodic and pseudo almost automorphic functions and applications to evolution equations. Appl. Anal. 89, 11-27 (2010)

6. Dads, EA, Ezzinbi, K: Existence of positive pseudo almost periodic solution for a class of functional equations arising in epidemic problems. Cybern. Syst. Anal. 30, 900-910 (1994)

7. Xu, B, Yuan, R: The existence of positive almost periodic type solutions for some neutral nonlinear integral equation. Nonlinear Anal., Theory Methods Appl. 60, 669-684 (2005)

8. Diagana, T: Pseudo Almost Periodic Functions in Banach Spaces. Nova Science Publishers, New York (2007)

9. Bochner, S: Abstrakte fastperiodische funktionen. Acta Math. 61, 149-184 (1933)

10. Sibuya, Y: Almost periodic solutions of Poisson's equation. Proc. Am. Math. Soc. 28, 195-198 (1971)

11. Sell, GR: Almost periodic solutions of linear partial differential equations. J. Math. Anal. Appl. 42, 302-312 (1973)

12. Zaidman, S: Almost Periodic Functions in Abstract Spaces. Pitman, Boston (1985)

13. Pankov, A: Bounded and Almost Periodic Solutions of Nonlinear Operator Differential Equations. Kluwer Academic, Dordrecht (1990). English Edition

14. Fournier, G, Szulkin, A, Willem, M: Semilinear elliptic equations in $\mathbb{R}^{n}$ with almost periodic or unbounded forcing term. SIAM J. Math. Anal. 27, 1653-1660 (1996)

15. N'Guérékata, GM, Pankov, A: Almost periodic elliptic equations: sub- and super-solutions. In: Operator Theory, Pseudo-Differential Equations, and Mathematical Physics. Operator Theory: Advances and Applications, vol. 228, pp. 275-292. Birkhäuser, Basel (2013)

16. Shubin, MA: Differential and pseudodifferential operators in spaces of almost periodic functions. Math. USSR Sb. 24(4), 547-573 (1974)

17. Shubin, MA: Almost periodic functions and partial differential operators. Russ. Math. Surv. 33(2), 1-52 (1978)

10.1186/1687-1847-2014-46

Cite this article as: Ji and Zhang: Pseudo almost periodic weak solutions of a semilinear elliptic equation. Advances in Difference Equations 2014, 2014:46

\section{Submit your manuscript to a SpringerOpen ${ }^{\ominus}$ journal and benefit from:}

- Convenient online submission

- Rigorous peer review

Immediate publication on acceptance

- Open access: articles freely available online

- High visibility within the field

- Retaining the copyright to your article 